Pacific

Journal of

Mathematics

TRANSFERENCE OF CERTAIN MAXIMAL HILBERT TRANSFORMS ON THE TORUS

Dashan Fan, HuOXIONG Wu and Fayou ZhaO 


\title{
TRANSFERENCE OF CERTAIN MAXIMAL HILBERT TRANSFORMS ON THE TORUS
}

\author{
DASHAN FAN, HUOXIONG WU AND FAYOU ZHAO
}

\begin{abstract}
Using transference techniques, we show that $L^{p}\left(\mathbb{R}^{n}\right)$ estimates for many operators may be transferred to the $L^{p}\left(\mathbb{T}^{n}\right)$ estimates on the $n$-torus $\mathbb{T}^{n}$ via measure-preserving actions of $\mathbb{R}^{n}$. These operators include the maximal bilinear Hilbert transform, the oscillation, and the variation and short variation operators of the Hilbert transform on the torus $\mathbb{T}$. As an extension, we study the (maximal) bilinear Riesz transforms on the $n$-torus $\mathbb{1}^{n}$.
\end{abstract}

\section{Introduction}

Let $\mathbb{C}$ be the complex plane and $\mathbb{R}_{+}^{2}$ the upper half plane

$$
\mathbb{R}_{+}^{2}=\{(x, y)=x+i y \in \mathbb{C}: y>0\} .
$$

The boundary of $\mathbb{R}_{+}^{2}$ is the real line $\mathbb{R}$. Consider the boundary condition $f \in L^{p}(\mathbb{R})$, where $f$ is real-valued and $1 \leq p<\infty$. It is well known that the Poisson integral

$$
u(x, y)=P_{y}(f)(x)=\frac{1}{\pi} \int_{\mathbb{R}} f(t) \frac{y}{(x-t)^{2}+y^{2}} d t
$$

is the solution of the Dirichlet problem on $\mathbb{R}_{+}^{2}$. Precisely, $u$ is a harmonic function on $\mathbb{R}_{+}^{2}$ and $u(x, y)$ tends to $f(x)$ nontangentially for almost all $x \in \mathbb{R}$ as $y \rightarrow 0^{+}$. There is a (unique) harmonic function

such that

$$
v(x, y)=Q_{y}(f)(x)=\frac{1}{\pi} \int_{\mathbb{R}} f(t) \frac{x-t}{(x-t)^{2}+y^{2}} d t
$$

$$
F(z)=u(x, y)+i v(x, y)
$$

is an analytic function on $\mathbb{R}_{+}^{2}$. This function $Q_{y}(f)(x)$ is called the conjugate Poisson integral of $f$. From [Stein and Weiss 1971, p. 186], we know that the

The research was supported by National Natural Science Foundation of China (Grant Nos. 11471288, 11471041, 11371295, 11201287), China Scholarship Council (No. 201406895019) and the FETU.

The corresponding author is Zhao.

MSC2010: primary 42B20, 42B25; secondary 42A50, 40A30.

Keywords: bilinear Hilbert transform, maximal operator, Riesz transform, oscillation, variation, torus, transference. 
function $F(z)$, with $z=x+i y$, has the nontangential limit $f(x)+\frac{i}{\pi} H f(x)$ for almost all $x \in \mathbb{R}$. Here $H$ is the Hilbert transform defined by

$$
H f(x)=\lim _{\varepsilon \rightarrow 0} H_{\varepsilon} f(x)
$$

and

$$
H_{\varepsilon} f(x)=\int_{|t|>\varepsilon} \frac{f(x-t)}{t} d t .
$$

To study the pointwise convergence of $H f(x)$, one then needs to study the truncated maximal Hilbert transform

$$
H^{*} f(x)=\sup _{\varepsilon>0}\left|H_{\varepsilon} f(x)\right| .
$$

Let

$$
D=\{z=x+i y \in \mathbb{C}:|z|<1\}
$$

be the unit disc. Its boundary $\mathbb{T}=\partial D$ is the one-dimensional torus. Without loss of generality, we may identify the torus $\mathbb{\mathbb { W }}$ with its fundamental interval $\left[-\frac{1}{2}, \frac{1}{2}\right)$. The Dirichlet problem on $D$ with the boundary condition $\tilde{f} \in L^{p}(\mathbb{T})$ similarly raises an analytic function $\widetilde{F}(z)=\tilde{u}(x, y)+i \tilde{v}(x, y)$. The function $\widetilde{F}(z)$, for $z=x+i y \in D$, has the nontangential limit $\tilde{f}(x)+\frac{i}{\pi} \widetilde{H} \tilde{f}(x)$ for almost all points $x \in \mathbb{T}$. Here $\widetilde{H}$ is the periodic version of the Hilbert transform defined by

$$
\tilde{H} \tilde{f}(x)=\lim _{\varepsilon \rightarrow 0} \widetilde{H}_{\varepsilon} \tilde{f}(x)
$$

and

$$
\widetilde{H}_{\varepsilon} \tilde{f}(x)=\int_{\varepsilon<|t|<\frac{1}{2}} \tilde{f}(x-t) \cot (\pi t) d t .
$$

By computing the Fourier coefficients, one can see that $\tilde{H} \tilde{f}(x)$ has the Fourier series

$$
\tilde{H} \tilde{f}(x)=\sum_{k=-\infty}^{\infty} i \operatorname{sgn}(k) a_{k} e^{2 \pi i k x}
$$

for any

$$
\tilde{f}(x)=\sum_{k=-\infty}^{\infty} a_{k} e^{2 \pi i k x}
$$

(see also [Edwards and Gaudry 1977]). It is known that $\sum_{k=-\infty}^{\infty} \operatorname{sgn}(k) a_{k} e^{2 \pi i k x}$ (up to a constant multiplier) is the conjugate Fourier series of $\tilde{f}$.

The bilinear Hilbert transform $\mathcal{H}(f, g)$ and the maximal bilinear Hilbert transform $\mathcal{H}^{*}(f, g)$ are defined respectively as

$$
\mathcal{H}(f, g)(x)=\lim _{\varepsilon \rightarrow 0} \int_{|t|>\varepsilon} \frac{f(x-t) g(x+t)}{t} d t
$$


and

$$
\mathcal{H}^{*}(f, g)(x)=\sup _{\varepsilon>0}\left|\int_{|t|>\varepsilon} \frac{f(x-t) g(x+t)}{t} d t\right| .
$$

The operator $\mathcal{H}(f, g)$ is not merely a formal extension from the Hilbert transform. It has deep roots in the study of certain harmonic analysis and PDE problems. The study of the bilinear Hilbert transform $\mathcal{H}(f, g)$ was initiated by Calderón when he studied certain Cauchy integrals $C_{\gamma}(f)$ along the Lipschitz curves. In order to obtain the $L^{2}$ boundedness of $C_{\gamma}(f)$, Calderón introduced a commutator (now known as the first Calderón commutator) and raised a famous conjecture, which says that $\mathcal{H}$ is a bounded operator from $L^{\infty} \times L^{2} \rightarrow L^{2}$; see [Jones 1994]. The conjecture was solved in a more general setting by Lacey and Thiele in their celebrated theorem:

Theorem A1 [Lacey and Thiele 1997]. Let $1<q, r \leq \infty$, and $\frac{2}{3}<p<\infty$. Then

$$
\|\mathcal{H}(f, g)\|_{L^{p}(\mathbb{R})} \preceq\|f\|_{L^{q}(\mathbb{R})}\|g\|_{L^{r}(\mathbb{R})},
$$

provided $\frac{1}{p}=\frac{1}{q}+\frac{1}{r}$.

The notation $A \preceq B$ for $A, B>0$ means that there exists a constant $c>0$ independent of all essential variables such that $A \leq c B$. We also use the notation $A \simeq B$ when $A \preceq B$ and $B \preceq A$.

The proof of the theorem by Lacey and Thiele involves a very elegant method of time-frequency analysis. The essence of the matter lies in their formulation and proof of certain almost orthogonal results on the phase space. Maximal forms of these results must be proved. These maximal inequalities rely in an essential way on a novel maximal inequality of Bourgain [1989; 1990]. By refining these maximal bilinear estimates and Bourgain's lemma, Lacey further obtained the following remarkable theorem:

Theorem A2 [Lacey 2000]. Let $1<q, r \leq \infty$ and $\frac{1}{p}=\frac{1}{q}+\frac{1}{r}$. If $\frac{2}{3}<p<\infty$, then

$$
\left\|\mathcal{H}^{*}(f, g)\right\|_{L^{p}(\mathbb{R})} \preceq\|f\|_{L^{q}(\mathbb{R})}\|g\|_{L^{r}(\mathbb{R})} .
$$

Based on Theorems A1 and A2, it is natural to expect to establish analogous theorems for the periodic bilinear Hilbert transform on the torus. Here, the bilinear Hilbert transform and its maximal operator on the torus are defined, initially on $C^{\infty}(\mathbb{T})$, by

$$
\tilde{\mathcal{H}}(\tilde{f}, \tilde{g})(x)=\text { p.v. } \int_{|t|<\frac{1}{2}} \tilde{f}(x-t) \tilde{g}(x+t) \cot (\pi t) d t
$$

and

$$
\widetilde{\mathcal{H}}^{*}(\tilde{f}, \tilde{g})(x)=\sup _{\varepsilon>0}\left|\int_{\varepsilon<|t|<\frac{1}{2}} \tilde{f}(x-t) \tilde{g}(x+t) \cot (\pi t) d t\right| .
$$


However, it seems quite difficult to adopt the time-frequency method used in [Lacey and Thiele 1997]. Thus, in [Fan and Sato 2001], the authors used a "transference" method to reduce the boundedness of $\tilde{\mathcal{H}}(\tilde{f}, \tilde{g})$ to the boundedness of $\mathcal{H}(f, g)$ by estimating an error term. The method of transference is a useful tool for obtaining norm estimates independent of the dimension for classical operators acting on $L^{p}\left(\mathbb{R}^{n}\right)$ (see [Auscher and Carro 1994; Blasco and Gillespie 2009; Coifman and Weiss 1977; Gillespie and Torrea 2004; Rubio de Francia 1989]). Fan and Sato [2001] proved the de Leeuw-type theorems (see [de Leeuw 1965]) for the transference of multilinear operators on Lebesgue spaces from $\mathbb{R}^{n}$ to the $n$-torus. In particular, they proved:

Theorem B. Let $1<q, r \leq \infty$ and $\frac{1}{p}=\frac{1}{q}+\frac{1}{r}$. If $\frac{2}{3}<p<\infty$, then

$$
\|\widetilde{\mathcal{H}}(\tilde{f}, \tilde{g})\|_{L^{p}(\mathbb{T})} \preceq\|\tilde{f}\|_{L^{q}(\mathbb{T})}\|\tilde{g}\|_{L^{r}(\mathbb{T})} .
$$

Note that

$$
\tilde{\mathcal{H}}(\tilde{f}, \tilde{g})(x) \simeq \sum_{k_{1} \in \mathbb{Z}} \sum_{k_{2} \in \mathbb{Z}} \operatorname{sgn}\left(k_{1}-k_{2}\right) a_{k_{1}} a_{k_{2}} e^{2 \pi i\left(k_{1}+k_{2}\right) x},
$$

where

$$
\tilde{f}(x)=\sum_{k_{1} \in \mathbb{Z}} a_{k_{1}} e^{2 \pi i k_{1} x} \quad \text { and } \quad \tilde{g}(x)=\sum_{k_{2} \in \mathbb{Z}} a_{k_{2}} e^{2 \pi i k_{2} x} .
$$

In [Fan and Sato 2001], the authors also studied the boundedness of the maximal multiplier operator

$$
\widetilde{T}^{* *}(\tilde{f}, \tilde{g})(x)=\sup _{\varepsilon>0}\left|\sum_{k_{1} \in \mathbb{Z}} \sum_{k_{2} \in \mathbb{Z}} m\left(\varepsilon k_{1}, \varepsilon k_{2}\right) a_{k_{1}} a_{k_{2}} e^{2 \pi i\left(k_{1}+k_{2}\right) x}\right|,
$$

where $m$ is a bounded and continuous function (see also [Berkson et al. 2006; 2007; Blasco et al. 2005; Grafakos and Honzík 2006] for transference methods on maximal bilinear operators). For the bilinear Hilbert transform, clearly we have

$$
\widetilde{\mathcal{H}}(\tilde{f}, \tilde{g})(x)=\widetilde{\mathcal{H}}^{* *}(\tilde{f}, \tilde{g})(x),
$$

since

$\sum_{k_{1} \in \mathbb{Z}} \sum_{k_{2} \in \mathbb{Z}} \operatorname{sgn}\left(\varepsilon k_{1}-\varepsilon k_{2}\right) a_{k_{1}} a_{k_{2}} e^{2 \pi i\left(k_{1}+k_{2}\right) x}=\sum_{k_{1} \in \mathbb{Z}} \sum_{k_{2} \in \mathbb{Z}} \operatorname{sgn}\left(k_{1}-k_{2}\right) a_{k_{1}} a_{k_{2}} e^{2 \pi i\left(k_{1}+k_{2}\right) x}$.

This observation indicates

$$
\widetilde{\mathcal{H}}^{*}(\tilde{f}, \tilde{g})(x) \neq \widetilde{\mathcal{H}}^{* *}(\tilde{f}, \tilde{g})(x) .
$$

Since the boundedness of $\widetilde{\mathcal{H}}^{*}(\tilde{f}, \tilde{g})$ still remains open, the first aim of this paper is to solve this problem by establishing the following analog of Lacey's theorem. 
Theorem 1.1. Let $1<q, r \leq \infty$ and $\frac{1}{p}=\frac{1}{q}+\frac{1}{r}$. If $\frac{2}{3}<p<\infty$, then

$$
\left\|\tilde{\mathcal{H}}^{*}(\tilde{f}, \tilde{g})\right\|_{L^{p}(\mathbb{T})} \preceq\|\tilde{f}\|_{L^{q}(\mathbb{T})}\|\tilde{g}\|_{L^{r}(\mathbb{T})} .
$$

We adopt the method in [Fan and Sato 2001] to prove the theorem, in which the main issue is to estimate error terms in order to reduce the boundedness of $L^{q}(\mathbb{\mathbb { T }}) \times L^{r}(\mathbb{\mathbb { T }}) \rightarrow L^{p}(\mathbb{T})$ to the known result for $L^{q}(\mathbb{R}) \times L^{r}(\mathbb{R}) \rightarrow L^{p}(\mathbb{R})$. This method additionally allows us to treat other operators related to the maximal Hilbert transform. Recall that the limits (1) and (3) mentioned above exist almost everywhere. Motivated by probability and ergodic theory [Bourgain 1989; Jones $1997 ; 1998]$, in order to obtain extra information on their convergence rate, as well as an estimate on the number of $\lambda$-jumps they can have, Campbell, Jones, Reinhold and Wierdl [Campbell et al. 2000] studied the oscillation and variation of the family $\left(H_{\varepsilon}\right)$ as $\varepsilon$ approaches 0 as follows.

For each fixed sequence $\left(t_{k}\right) \searrow 0$, define the oscillation and variation operators by

$$
\begin{aligned}
\mathscr{O}\left(H_{*} f\right)(x) & =\left(\sum_{k=1}^{\infty} \sup _{t_{k+1} \leq \varepsilon_{k+1}<\varepsilon_{k} \leq t_{k}}\left|H_{\varepsilon_{k}} f(x)-H_{\varepsilon_{k+1}} f(x)\right|^{2}\right)^{\frac{1}{2}}, \\
\mathscr{V}_{\varrho}\left(H_{*} f\right)(x) & =\sup _{\left(\varepsilon_{k}\right) \searrow 0}\left(\sum_{k=1}^{\infty}\left|H_{\varepsilon_{k}} f(x)-H_{\varepsilon_{k+1}} f(x)\right|^{\varrho}\right)^{\frac{1}{\varrho}},
\end{aligned}
$$

respectively. Also, define

$$
V_{k}\left(H_{*} f\right)(x)=\sup _{\left(\varepsilon_{j}\right) \searrow 0}\left(\sum_{\frac{1}{2^{k}}<\varepsilon_{j+1}<\varepsilon_{j} \leq \frac{1}{2^{k-1}}}\left|H_{\varepsilon_{j}} f(x)-H_{\varepsilon_{j+1}} f(x)\right|^{2}\right)^{\frac{1}{2}},
$$

where the supremum is taken over all decreasing sequences $\left(\varepsilon_{j}\right)$. Then the "short variation operator" is defined by

$$
S_{V}\left(H_{*} f\right)(x)=\left(\sum_{k=-\infty}^{\infty} V_{k}\left(H_{*} f(x)\right)^{2}\right)^{\frac{1}{2}} .
$$

For convenience, all the integrals are defined on the Schwartz class.

We recall the following results from [Campbell et al. 2000].

Theorem C. The oscillation operator $\mathscr{O}\left(H_{*}\right)$ satisfies

$$
\left\|\mathscr{O}\left(H_{*} f\right)\right\|_{L^{p}(\mathbb{R})} \leq c_{p}\|f\|_{L^{p}(\mathbb{R})}
$$

for $1<p<\infty$ and $\left|\left\{x \in \mathbb{R}: \mathscr{O}\left(H_{*} f\right)(x)>\lambda\right\}\right| \leq(c / \lambda)\|f\|_{L^{1}(\mathbb{R})}$.

Theorem D. If $\varrho>2$, then the variation operator $\mathscr{V}_{\varrho}\left(H_{*}\right)$ satisfies

$$
\left\|\mathscr{V}_{\varrho}\left(H_{*} f\right)\right\|_{L^{p}(\mathbb{R})} \leq c(p, \varrho)\|f\|_{L^{p}(\mathbb{R})}
$$

for $1<p<\infty$ and $\left|\left\{x \in \mathbb{R}: \mathscr{V}_{\varrho}\left(H_{*} f\right)(x)>\lambda\right\}\right| \leq(c(\varrho) / \lambda)\|f\|_{L^{1}(\mathbb{R})}$. 
Theorem E. The short variation operator $S_{V}\left(H_{*}\right)$ satisfies

$$
\left\|S_{V}\left(H_{*} f\right)\right\|_{L^{p}(\mathbb{R})} \leq c_{p}\|f\|_{L^{p}(\mathbb{R})}
$$

for $1<p<\infty$ and $\left|\left\{x \in \mathbb{R}: S_{V}\left(H_{*} f\right)(x)>\lambda\right\}\right| \leq(c / \lambda)\|f\|_{L^{1}(\mathbb{R})}$.

Our second aim is to use the transference method to study the analogs of these operators on the torus. For each fixed sequence $\left(t_{k}\right) \searrow 0$, define the oscillation and variation operators on the torus by

$$
\begin{aligned}
& \mathscr{O}\left(\widetilde{H}_{*} \tilde{f}\right)(x)=\left(\sum_{k=1}^{\infty} \sup _{t_{k+1} \leq \varepsilon_{k+1}<\varepsilon_{k} \leq t_{k}}\left|\widetilde{H}_{\varepsilon_{k}} \tilde{f}(x)-\widetilde{H}_{\varepsilon_{k+1}} \tilde{f}(x)\right|^{2}\right)^{\frac{1}{2}}, \\
& \mathscr{V}_{\varrho}\left(\widetilde{H}_{*} \tilde{f}\right)(x)=\sup _{\left(\varepsilon_{k}\right) \searrow 0}\left(\sum_{k=1}^{\infty}\left|\widetilde{H}_{\varepsilon_{k}} \tilde{f}(x)-\widetilde{H}_{\varepsilon_{k+1}} \tilde{f}(x)\right|^{\varrho}\right)^{\frac{1}{\varrho}},
\end{aligned}
$$

respectively. Also, define the operator $V_{k}\left(\widetilde{H}_{*}\right)$ on the torus by

$$
V_{k}\left(\widetilde{H}_{*} \tilde{f}\right)(x)=\sup _{\left(\varepsilon_{j}\right) \searrow 0}\left(\sum_{\frac{1}{2^{k}}<\varepsilon_{j+1}<\varepsilon_{j} \leq \frac{1}{2^{k-1}}}\left|\widetilde{H}_{\varepsilon_{j}} \tilde{f}(x)-\widetilde{H}_{\varepsilon_{j+1}} \tilde{f}(x)\right|^{2}\right)^{\frac{1}{2}},
$$

where the supremum is taken over all decreasing sequences $\left(\varepsilon_{j}\right)$. Then define the "short variation operator" on the torus by

$$
S_{V}\left(\widetilde{H}_{*} \tilde{f}\right)(x)=\left(\sum_{k=-\infty}^{\infty}\left(V_{k}\left(\widetilde{H}_{*} \tilde{f}(x)\right)^{2}\right)^{\frac{1}{2}} .\right.
$$

For simplicity, we define these operators on the space $C^{\infty}(\mathbb{T})$.

We establish the following theorems.

Theorem 1.2. The oscillation operator $\mathscr{O}\left(\widetilde{H}_{*}\right)$ satisfies

$$
\left\|\mathscr{O}\left(\tilde{H}_{*} \tilde{f}\right)\right\|_{L^{p}(\mathbb{T})} \leq c_{p}\|\tilde{f}\|_{L^{p}(\mathbb{T})}
$$

for $1<p<\infty$ and $\left|\left\{x \in \mathbb{T}: \mathscr{O}\left(\tilde{H}_{*} \tilde{f}\right)(x)>\lambda\right\}\right| \leq(c / \lambda)\|\tilde{f}\|_{L^{1}(\mathbb{T})}$.

Theorem 1.3. If $\varrho>2$, then the variation operator $\mathscr{V}_{\varrho}\left(\widetilde{H}_{*}\right)$ satisfies

$$
\left\|\mathscr{V}_{\varrho}\left(\widetilde{H}_{*} \tilde{f}\right)\right\|_{L^{p}(\mathbb{T})} \leq c(p, \varrho)\|\tilde{f}\|_{L^{p}(\mathbb{T})}
$$

for $1<p<\infty$ and $\left|\left\{x \in \mathbb{T}: \mathscr{V}_{\varrho}\left(\widetilde{H}_{*} \tilde{f}\right)(x)>\lambda\right\}\right| \leq(c(\varrho) / \lambda)\|\tilde{f}\|_{L^{1}(\mathbb{T})}$.

Theorem 1.4. The short variation operator $S_{V}\left(\widetilde{H}_{*}\right)$ satisfies

$$
\left\|S_{V}\left(\tilde{H}_{*} \tilde{f}\right)\right\|_{L^{p}(\mathbb{T})} \leq c_{p}\|\tilde{f}\|_{L^{p}(\mathbb{T})}
$$

for $1<p<\infty$ and $\left|\left\{x \in \mathbb{T}: S_{V}\left(\widetilde{H}_{*} \tilde{f}\right)(x)>\lambda\right\}\right| \leq(c / \lambda)\|\tilde{f}\|_{L^{1}(\mathbb{T})}$. 
As we mentioned, the method in [Fan and Sato 2001] shows that $L^{p}\left(\mathbb{R}^{n}\right)$ estimates for many linear operators may be transferred to their corresponding $L^{p}$ estimates on the torus via measure-preserving actions of $\mathbb{R}^{n}$. As a further application and extension, we consider the bilinear Riesz transform on the $n$-torus. Recall that the bilinear singular integral with rough kernel on $\mathbb{R}^{n}$ is defined by

$$
T_{\Omega}(f, g)(x)=\lim _{\varepsilon \rightarrow 0} \int_{|y|>\varepsilon} f(x-y) g(x+y) \frac{\Omega\left(y^{\prime}\right)}{|y|^{n}} d y,
$$

where $\Omega\left(y^{\prime}\right)$ is a function defined on the unit sphere $S^{n-1}$ in Euclidean space $\mathbb{R}^{n}$ and whose integral over $S^{n-1}$ is zero. One then obtains the bilinear Riesz transform by taking $\Omega(x)=x_{j} /|x|$, where $x_{j}$ is the $j$-th component of $x$. Using the same transference method, we also can transfer the $L^{p}$-boundedness of the maximal bilinear Riesz transform from $\mathbb{R}^{n}$ to $\mathbb{T}^{n}$. This fact is discussed in the last two sections.

Throughout this article, we use the letter $c$ to denote a positive constant, which is independent of the main parameters and not necessarily the same at each occurrence.

\section{Proof of Theorems 1.1-1.4}

In this section we give the proof of Theorems 1.1-1.4. As is well known, Euler discovered two remarkable expressions for circular functions, one as an infinite product and the other as an infinite series. For the sine function he established the formula

$$
\sin (\pi x)=\pi x \prod_{k=1}^{\infty}\left(1-\frac{x^{2}}{k}\right)=\pi x \prod_{k \in \mathbb{Z} \backslash\{0\}}\left(1+\frac{x}{k}\right)
$$

(see [Varadarajan 2007]). By logarithmic differentiation one obtains

$$
\cot (\pi x)=\text { p.v. } \frac{1}{\pi} \sum_{k \in \mathbb{Z}}\left(\frac{1}{x+k}\right),
$$

where p.v. means the Cauchy principal value, that is, that the sum has to be interpreted as the limit

$$
\lim _{N \rightarrow+\infty} \sum_{k=-N}^{N} \frac{1}{x+k}=\frac{1}{x}+\sum_{k=1}^{\infty}\left(\frac{1}{x+k}-\frac{1}{x-k}\right), \quad N \in \mathbb{Z}^{+} .
$$

Let $\chi_{A}(t)$ be the characteristic function of the set $A=\{t \in \mathbb{R}:|t|>1\}$. To prove Theorem 1.1, we need the following lemma.

Lemma 2.1. For $\varepsilon<|t| \leq \frac{1}{2}$, we have

$$
\cot (\pi t) \chi_{A}\left(\frac{t}{\varepsilon}\right)=\frac{1}{\pi} \sum_{k \in \mathbb{Z}} \frac{1}{t+k} \chi_{A}\left(\frac{t+k}{\varepsilon}\right)-\frac{1}{\pi} \sum_{k \in \mathbb{Z} \backslash\{0\}} \frac{1}{t+k} \chi_{A^{C}}\left(\frac{t}{\varepsilon}\right)
$$


and the estimate

$$
\left|\sum_{k \in \mathbb{Z} \backslash\{0\}} \frac{1}{t+k} \chi_{A^{C}}\left(\frac{t}{\varepsilon}\right)\right| \preceq|t| \chi_{A^{C}}\left(\frac{t}{\varepsilon}\right),
$$

where $A^{C}$ is the complement of the set $A$.

Proof. Using (8), we write the term $\cot (\pi t) \chi_{A}(t / \varepsilon)$ as

$$
\begin{aligned}
\cot (\pi t) \chi_{A}\left(\frac{t}{\varepsilon}\right) & =\frac{1}{\pi} \sum_{k \in \mathbb{Z}} \frac{1}{t+k} \chi_{A}\left(\frac{t}{\varepsilon}\right) \\
& =\frac{1}{\pi} \sum_{k \in \mathbb{Z} \backslash\{0\}} \frac{1}{t+k} \chi_{A}\left(\frac{t}{\varepsilon}\right)+\frac{1}{\pi t} \chi_{A}\left(\frac{t}{\varepsilon}\right) .
\end{aligned}
$$

Since $\chi_{A}$ is the characteristic function of the set $\{t \in \mathbb{R}:|t|>1\}$, it is easy to see that for $\varepsilon<|t| \leq \frac{1}{2}$ and $k \in \mathbb{Z} \backslash\{0\}$, we have

$$
\chi_{A}\left(\frac{t+k}{\varepsilon}\right)=1 .
$$

The fact above leads to

$$
\frac{1}{\pi t} \chi_{A}\left(\frac{t}{\varepsilon}\right)=\frac{1}{\pi t} \chi_{A}\left(\frac{t}{\varepsilon}\right)+\frac{1}{\pi} \sum_{k \in \mathbb{Z} \backslash\{0\}} \frac{1}{t+k} \chi_{A}\left(\frac{t+k}{\varepsilon}\right)-\frac{1}{\pi} \sum_{k \in \mathbb{Z} \backslash\{0\}} \frac{1}{t+k} .
$$

Hence we have

$$
\cot (\pi t) \chi_{A}\left(\frac{t}{\varepsilon}\right)=\frac{1}{\pi} \sum_{k \in \mathbb{Z}} \frac{1}{t+k} \chi_{A}\left(\frac{t+k}{\varepsilon}\right)-\frac{1}{\pi} \sum_{k \in \mathbb{Z} \backslash\{0\}} \frac{1}{t+k} \chi_{A^{c}}\left(\frac{t}{\varepsilon}\right) .
$$

It now remains to estimate

$$
\sum_{k \in \mathbb{Z} \backslash\{0\}} \frac{1}{t+k} \chi_{A^{C}}\left(\frac{t}{\varepsilon}\right)
$$

From (9), we know

$$
\sum_{k \in \mathbb{Z} \backslash\{0\}} \frac{1}{t+k} \chi_{A^{C}}\left(\frac{t}{\varepsilon}\right)=\sum_{k=1}^{\infty}\left(\frac{1}{t+k}+\frac{1}{t-k}\right) \chi_{A^{C}}\left(\frac{t}{\varepsilon}\right)=2 t \sum_{k=1}^{\infty} \frac{1}{t^{2}-k^{2}} \chi_{A^{C}}\left(\frac{t}{\varepsilon}\right) .
$$

Using this yields

$$
\left|\sum_{k \in \mathbb{Z} \backslash\{0\}} \frac{1}{t+k} \chi_{A^{C}}\left(\frac{t}{\varepsilon}\right)\right| \preceq|t| \chi_{A^{C}}\left(\frac{t}{\varepsilon}\right),
$$

which completes the proof. 
Proof of Theorem 1.1. For simplicity, we introduce some notation. Denote by

$$
\begin{aligned}
\mathfrak{R}(t) & =\frac{\chi_{A}(t)}{t}, & \mathfrak{R}_{\varepsilon}(t) & =\frac{1}{\varepsilon} \mathfrak{R}\left(\frac{t}{\varepsilon}\right), \\
\widetilde{\mathfrak{R}}_{\varepsilon}(t) & =\frac{1}{\varepsilon} \sum_{k \in \mathbb{Z}} \mathfrak{R}\left(\frac{t+k}{\varepsilon}\right), & r_{\varepsilon}(t) & =\sum_{k \in \mathbb{Z} \backslash\{0\}} \frac{1}{t+k} \chi_{A^{C}}\left(\frac{t}{\varepsilon}\right) .
\end{aligned}
$$

Then we have

$$
\mathcal{H}_{\varepsilon}(f, g)(x)=\int_{\mathbb{R}} f(x-t) g(x+t) \mathfrak{R}_{\varepsilon}(t) d t .
$$

Let

$$
\widetilde{\mathbb{M}}_{\varepsilon}(\tilde{f}, \tilde{g})(x)=\int_{|t|<\frac{1}{2}} \tilde{f}(x-t) \tilde{g}(x+t) \widetilde{\mathfrak{R}}_{\varepsilon}(t) d t .
$$

Because of Lemma 2.1, one has

$$
\widetilde{H}^{*}(\tilde{f}, \tilde{g})(x) \leq \frac{1}{\pi} \sup _{\varepsilon>0}\left|\widetilde{\mathbb{M}}_{\varepsilon}(\tilde{f}, \tilde{g})(x)\right|+\frac{1}{\pi} M(\tilde{f}, \tilde{g})(x),
$$

where

$$
M(\tilde{f}, \tilde{g})(x)=\sup _{\varepsilon>0}\left|\int_{|t|<\frac{1}{2}} \tilde{f}(x-t) \tilde{g}(x+t) r_{\varepsilon}(t) d t\right| .
$$

By Lemma 2.1, the Minkowski integral inequality and Hölder's inequality, we see that, for $p \geq 1$,

$$
\begin{aligned}
\|M(\tilde{f}, \tilde{g})\|_{L^{p}(\mathbb{T})} & \preceq \int_{-\frac{1}{2}}^{\frac{1}{2}}\|\tilde{f}(x-t) \tilde{g}(x+t)\|_{L^{p}(\mathbb{T}, d x)}|t| d t \\
& \preceq\|\tilde{f}\|_{L^{q}(\mathbb{\mathbb { T }})}\|\tilde{g}\|_{L^{r}(\mathbb{\mathbb { T }})} .
\end{aligned}
$$

On the other hand, using Hölder's inequality, we have

$$
\begin{aligned}
\left\|\int_{-\frac{1}{2}}^{\frac{1}{2}} \tilde{f}(x-t) \tilde{g}(x+t) r_{\varepsilon}(t) d t\right\|_{L^{1 / 2}(\mathbb{T})} & \preceq\left\|\int_{-\frac{1}{2}}^{\frac{1}{2}}\left|\tilde{f}(x-t) \tilde{g}(x+t) r_{\varepsilon}(t)\right| d t\right\|_{L^{1}(\mathbb{T})} \\
& \preceq\|\tilde{f}\|_{L^{1}(\mathbb{T})}\|\tilde{g}\|_{L^{1}(\mathbb{T})} .
\end{aligned}
$$

Then an interpolation yields that, for all $\frac{1}{2} \leq p<\infty$,

$$
\|M(\tilde{f}, \tilde{g})\|_{L^{p}(\mathbb{T})} \preceq\|\tilde{f}\|_{L^{q}(\mathbb{T})}\|\tilde{g}\|_{L^{r}(\mathbb{T})} .
$$

Thus, to prove the theorem, we only need to show that

$$
\left\|\sup _{\varepsilon>0}\left|\tilde{\mathbb{M}}_{\varepsilon}(\tilde{f}, \tilde{g})\right|\right\|_{L^{p}(\mathbb{T})} \preceq\|\tilde{f}\|_{L^{q}(\mathbb{T})}\|\tilde{g}\|_{L^{r}(\mathbb{T})} .
$$


It is easy to compute that the Fourier coefficients of $\widetilde{\mathfrak{R}}_{\varepsilon}(t)$ are

$$
\begin{aligned}
c_{m, \varepsilon} & =\int_{-\frac{1}{2}}^{\frac{1}{2}} \sum_{k \in \mathbb{Z}} \frac{1}{\varepsilon} \mathfrak{R}\left(\frac{t+k}{\varepsilon}\right) e^{-i 2 \pi m t} d t \\
& =\int_{\mathbb{R}} \mathfrak{R}(t) e^{-i 2 \pi m t \varepsilon} d t=-i \int_{|t|>1} \frac{\sin (2 \pi m t \varepsilon)}{t} d t \\
& =-\pi i \operatorname{sgn}(\varepsilon m)+i \int_{|t| \leq 2 \pi} \frac{\sin (\varepsilon m t)}{t} d t .
\end{aligned}
$$

Clearly, $c_{m, \varepsilon}$ is uniformly bounded on $\varepsilon>0$ and $m \in \mathbb{Z}$. On the other hand, it is not difficult to check that

$$
\int_{|t|<\frac{1}{2}} \tilde{f}(x-t) \tilde{g}(x+t) \widetilde{\mathfrak{R}}_{\varepsilon}(t) d t=\sum_{m=-\infty}^{\infty} \sum_{n=-\infty}^{\infty} a_{m} b_{n} c_{m-n, \varepsilon} e^{2 \pi i(n+m) x},
$$

where

$$
\tilde{f}(x)=\sum_{m=-\infty}^{\infty} a_{m} e^{2 \pi i m x} \text { and } \quad \tilde{g}(x)=\sum_{n=-\infty}^{\infty} b_{n} e^{2 \pi i n x} .
$$

Now pick $\Psi \in \mathscr{S}(\mathbb{R})$ satisfying $\Psi(x)=1$ on $\left[-\frac{1}{2}, \frac{1}{2}\right], \operatorname{supp}(\Psi) \subset\left[-\frac{3}{4}, \frac{3}{4}\right]$ and $0 \leq \Psi(x) \leq 1$. For any positive $N$, denote the function $\Psi^{N}$ by

$$
\Psi^{N}(x)=\Psi(x / N) .
$$

Consider the error term given by

$$
E_{N, \varepsilon}(\tilde{f}, \tilde{g})(x)=\Psi(x / N)^{2} \widetilde{\mathbb{H}}_{\varepsilon}(\tilde{f}, \tilde{g})(x)-\mathcal{H}_{\varepsilon}\left(\Psi^{N} \tilde{f}, \Psi^{N} \tilde{g}\right)(x) .
$$

The error term $E_{N, \varepsilon}(\tilde{f}, \tilde{g})(x)$ roughly gives the difference of $\mathcal{H}_{\varepsilon}$ on $\mathbb{R}$ and $\widetilde{\mathbb{H}}_{\varepsilon}$ on the torus. By checking the Fourier transform, we have

$$
\begin{aligned}
\mathcal{H}_{\varepsilon}\left(\Psi^{N} \tilde{f}, \Psi^{N} \tilde{g}\right)(x)=\sum_{m=-\infty}^{\infty} \sum_{n=-\infty}^{\infty} a_{m} b_{n} e^{2 \pi i(n+m) x} & \\
& \times \int_{\mathbb{R}} \mathfrak{R}_{\varepsilon}(t) \Psi\left(\frac{x+t}{N}\right) \Psi\left(\frac{x-t}{N}\right) e^{2 \pi i(n-m) t} d t .
\end{aligned}
$$

The definition of the inverse Fourier transform on the space of Schwartz functions shows that

$$
\begin{aligned}
\int_{\mathbb{R}} \mathfrak{R}_{\varepsilon}(t) \Psi\left(\frac{x+t}{N}\right) \Psi\left(\frac{x-t}{N}\right) e^{2 \pi i(n-m) t} d t \\
=\int_{\mathbb{R}} \Re_{\varepsilon}(t) \int_{\mathbb{R}} \int_{\mathbb{R}} \widehat{\Psi}(u) \widehat{\Psi}(v) e^{2 \pi i u(x+t) / N} e^{2 \pi i v(x-t) / N} d u d v e^{2 \pi i(n-m) t} d t \\
=\int_{\mathbb{R}} \int_{\mathbb{R}} \widehat{\Psi}(u) \widehat{\Psi}(v) e^{2 \pi i x(u+v) / N}\left(\int_{\mathbb{R}} \mathfrak{R}_{\varepsilon}(t) e^{2 \pi i(u-v) t / N} e^{2 \pi i(n-m) t} d t\right) d u d v .
\end{aligned}
$$


Therefore, we obtain that

$$
\begin{aligned}
E_{N, \varepsilon}(\tilde{f}, \tilde{g})(x)= & \sum_{m=-\infty}^{\infty} \sum_{n=-\infty}^{\infty} a_{m} b_{n} e^{2 \pi i(n+m) x} \\
& \times \int_{\mathbb{R}} \int_{\mathbb{R}} \widehat{\Psi}(u) \widehat{\Psi}(v)\left(c_{m-n, \varepsilon}-c_{m-n+(v-u) / N, \varepsilon}\right) e^{2 \pi i x(u+v) / N} d u d v .
\end{aligned}
$$

If $m=n$, then

$$
\begin{aligned}
\int_{\mathbb{R}} \int_{\mathbb{R}} \widehat{\Psi}(u) \widehat{\Psi}(v)\left(c_{m-n, \varepsilon}-c_{m-n+(v-u) / N, \varepsilon}\right) e^{2 \pi i x(u+v) / N} d u d v \\
=i \int_{\mathbb{R}} \int_{u>v} \widehat{\Psi}(u) \widehat{\Psi}(v)\left(\int_{|t|>1} \frac{\sin \varepsilon 2 \pi t(v-u) / N}{t} d t\right) e^{2 \pi i x(u+v) / N} d u d v \\
\quad-i \int_{\mathbb{R}} \int_{v>u} \widehat{\Psi}(u) \widehat{\Psi}(v)\left(\int_{|t|>1} \frac{\sin \varepsilon 2 \pi t(u-v) / N}{t} d t\right) e^{2 \pi i x(u+v) / N} d u d v \\
=0 .
\end{aligned}
$$

If $m \neq n$, for any sufficiently small $\delta>0$, we choose an $L>0$ such that

$$
\left|\int_{u^{2}+v^{2}>L} \widehat{\Psi}(u) \widehat{\Psi}(v)\left(c_{m-n, \varepsilon}-c_{m-n+(v-u) / N, \varepsilon}\right) e^{2 \pi i x(u+v) / N} d u d v\right|<\delta .
$$

Now we let $N$ be sufficiently large so that, for $u^{2}+v^{2} \leq L$,

$$
\operatorname{sgn}(m-n)=\operatorname{sgn}(m-n+(v-u) / N) .
$$

By this choice, for all $0<\varepsilon<\frac{1}{2}$, we have

$$
\begin{aligned}
& \left|\int_{u^{2}+v^{2} \leq L} \widehat{\Psi}(u) \widehat{\Psi}(v)\left(c_{m-n, \varepsilon}-c_{m-n+(v-u) / N, \varepsilon}\right) e^{2 \pi i x(u+v) / N} d u d v\right| \\
& \preceq \int_{u^{2}+v^{2} \leq L}\left|\widehat{\Psi}(u) \widehat{\Psi}(v) \int_{|t|<2 \pi} \frac{\sin (\varepsilon t(m-n))-\sin (\varepsilon t(m-n+(v-u) / N))}{t} d t\right| d u d v \\
& =o(1) \text { as } N \rightarrow \infty .
\end{aligned}
$$

Since $\left(a_{n}\right)$ and $\left(b_{m}\right)$ are rapidly decreasing sequences, it is easy to see that

$$
\lim _{N \rightarrow \infty} \sup _{0<\varepsilon<1 / 2}\left|E_{N, \varepsilon}(\tilde{f}, \tilde{g})(x)\right|=0 .
$$

Applying that

$$
\sup _{0<\varepsilon<1 / 2}\left|\widetilde{\mathbb{H}}_{\varepsilon}(\tilde{f}, \tilde{g})(x)\right|
$$

is a periodic function, together with Theorem A2, we now have, as $N \rightarrow \infty$, 


$$
\begin{aligned}
& \left\|\sup _{0<\varepsilon<1 / 2}\left|\widetilde{\mathbb{H}}_{\varepsilon}(\tilde{f}, \tilde{g})\right|\right\|_{L^{p}(\mathbb{T})}=\left(\int_{-\frac{1}{2}}^{\frac{1}{2}}\left(\sup _{0<\varepsilon<1 / 2}\left|\widetilde{\mathbb{H}_{\varepsilon}}(\tilde{f}, \tilde{g})(x)\right|\right)^{p} d x\right)^{\frac{1}{p}} \\
& =\frac{1}{N^{1 / p}}\left(\int_{-\frac{N}{2}}^{\frac{N}{2}} \sup _{0<\varepsilon<1 / 2} \Psi(x / N)^{2}\left|\widetilde{\mathbb{H}}_{\varepsilon}(\tilde{f}, \tilde{g})(x)\right|^{p} d x\right)^{\frac{1}{p}} \\
& \preceq o(1)+\frac{1}{N^{1 / p}}\left(\int_{\mathbb{R}} \sup _{0<\varepsilon<1 / 2}\left|\mathcal{H}_{\varepsilon}\left(\Psi^{N} \tilde{f}, \Psi^{N} \tilde{g}\right)(x)\right|^{p} d x\right)^{\frac{1}{p}} \\
& \preceq o(1)+\frac{1}{N^{1 / p}}\left\|\Psi^{N} \tilde{f}\right\|_{L^{q}(\mathbb{R})}\left\|\Psi^{N} \tilde{g}\right\|_{L^{r}(\mathbb{R})} \\
& \preceq o(1)+\|\tilde{f}\|_{L^{q}(\mathbb{T})}\|\tilde{g}\|_{L^{r}(\mathbb{T})} .
\end{aligned}
$$

Thus we get the desired result by letting $N \rightarrow \infty$.

We again will use the transference method to prove Theorems 1.2-1.4. To this end, we need a key lemma in order to estimate error terms.

Lemma 2.2. Let $\mathfrak{R}_{\varepsilon}, \widetilde{\mathfrak{R}}_{\varepsilon}$ and $\Psi$ be as defined in (10), (11) and (13), respectively. For $k \in \mathbb{Z}^{+}$, set

$$
\begin{aligned}
& \widetilde{H}_{\varepsilon_{k}, \varepsilon_{k+1}}(\tilde{f})(x)=\left(\widetilde{\mathfrak{R}}_{\varepsilon_{k}}-\widetilde{\mathfrak{R}}_{\varepsilon_{k+1}}\right) * \tilde{f}(x), \\
& H_{\varepsilon_{k}, \varepsilon_{k+1}}(f)(x)=\left(\mathfrak{R}_{\varepsilon_{k}}-\mathfrak{R}_{\varepsilon_{k+1}}\right) * f(x) .
\end{aligned}
$$

For fixed $N \in \mathbb{Z}^{+}$, define the error term

$$
E_{N, \varepsilon_{k}, \varepsilon_{k+1}}(\tilde{f})(x)=\Psi^{N}(x) \widetilde{H}_{\varepsilon_{k}, \varepsilon_{k+1}}(\tilde{f})(x)-H_{\varepsilon_{k}, \varepsilon_{k+1}}\left(\Psi^{N} \tilde{f}\right)(x) .
$$

Let $1 \leq p<\infty$. As $N \rightarrow \infty$, we have:

(i) For each fixed sequence $\left(t_{k}\right) \searrow 0$,

$$
\left\|\left(\sum_{k=1}^{\infty} \sup _{t_{k+1} \leq \varepsilon_{k+1}<\varepsilon_{k} \leq t_{k}}\left|E_{N, \varepsilon_{k}, \varepsilon_{k+1}}(\tilde{f})(x)\right|^{2}\right)^{\frac{1}{2}}\right\|_{L^{p}(\mathbb{T})}=o(1) .
$$

(ii) If $\frac{1}{2^{k}} \leq \varepsilon_{k+1}<\varepsilon_{k} \leq \frac{1}{2^{k-1}}$, then for $\varrho>2$,

$$
\left\|\sup _{\left(\varepsilon_{k}\right) \searrow 0}\left(\sum_{k=1}^{\infty}\left|E_{N, \varepsilon_{k}, \varepsilon_{k+1}}(\tilde{f})(x)\right|^{\varrho}\right)^{\frac{1}{\varrho}}\right\|_{L^{p}(\mathbb{\mathbb { T }})}=o(1) .
$$

Proof. By the previous calculation (12), we know that the Fourier coefficients of $\widetilde{\mathfrak{R}}_{\varepsilon}(t)$ are

$$
c_{l, \varepsilon}=\int_{\mathbb{R}} \mathfrak{R}(t) e^{-i \varepsilon 2 \pi \ell t} d t=-i \int_{|t|>1} \frac{\sin (\varepsilon 2 \pi \ell t)}{t} d t, \quad \ell \in \mathbb{Z} .
$$


Note that

$$
\int_{|t|<\frac{1}{2}} \widetilde{\mathfrak{R}}_{\varepsilon_{k}}(t) \tilde{f}(x-t) d t=\sum_{m=-\infty}^{\infty} a_{m} c_{m, \varepsilon_{k}} e^{2 \pi i m x} .
$$

For fixed $N \in \mathbb{Z}^{+}$, we have

$$
\Psi(x / N) \widetilde{H}_{\varepsilon_{k}, \varepsilon_{k+1}}(\tilde{f})(x)=\sum_{m=-\infty}^{\infty} a_{m} e^{2 \pi i m x} \int_{\mathbb{R}} \widehat{\Psi}(u)\left(c_{m, \varepsilon_{k}}-c_{m, \varepsilon_{k+1}}\right) e^{2 \pi u x / N} d u .
$$

Also, by a similar estimate as in Theorem 1.1, we obtain

$$
H_{\varepsilon_{k}, \varepsilon_{k+1}}\left(\Psi^{N} \tilde{f}\right)(x)=\sum_{m=-\infty}^{\infty} a_{m} e^{2 \pi i m x} \int_{\mathbb{R}} \widehat{\Psi}(u)\left(c_{m+u / N, \varepsilon_{k}}-c_{m+u / N, \varepsilon_{k+1}}\right) e^{2 \pi u x / N} d u .
$$

Consequently,

$$
\begin{aligned}
E_{N, \varepsilon_{k}, \varepsilon_{k+1}}(\tilde{f})(x) & =\sum_{m=-\infty}^{\infty} a_{m} e^{2 \pi i m x} \\
& \times \int_{\mathbb{R}} \widehat{\Psi}(u)\left(\left(c_{m, \varepsilon_{k}}-c_{m, \varepsilon_{k+1}}\right)-\left(c_{m+u / N, \varepsilon_{k}}-c_{m+u / N, \varepsilon_{k+1}}\right)\right) e^{2 \pi u x / N} d u .
\end{aligned}
$$

In order to simplify the notation, we denote by $C_{N}(u)$ the term

$$
\left(c_{m, \varepsilon_{k}}-c_{m, \varepsilon_{k+1}}\right)-\left(c_{m+u / N, \varepsilon_{k}}-c_{m+u / N, \varepsilon_{k+1}}\right) .
$$

To evaluate the inner integral above, we first deal with the term $C_{N}(u)$. From the second expression of (14),

$$
C_{N}(u)=2 i \int_{\varepsilon_{k+1}}^{\varepsilon_{k}} \frac{\sin (2 \pi m t)-\sin (2 \pi(m+u / N) t)}{t} d t
$$

We consider two cases: $m=0$ and $m \neq 0$.

If $m=0$, one has

$$
\left|C_{N}(u)\right|=\left|-2 i \int_{\varepsilon_{k+1}}^{\varepsilon_{k}} \frac{\sin (2 \pi t u / N)}{t} d t\right| \leq 4 \pi|u| \frac{1}{N}\left(\varepsilon_{k}-\varepsilon_{k+1}\right) .
$$

If $m \neq 0$, it follows from trigonometric identities that

$$
\left|C_{N}(u)\right| \leq 2 \int_{\varepsilon_{k+1}}^{\varepsilon_{k}} \frac{|\sin (\pi t u / N)|}{t} d t \leq 2 \pi|u| \frac{1}{N}\left(\varepsilon_{k}-\varepsilon_{k+1}\right) .
$$


Applying the above estimates, we have

$$
\begin{aligned}
\left\|\left(\sum_{k=1}^{\infty} \sup _{t_{k+1} \leq \varepsilon_{k+1}<\varepsilon_{k} \leq t_{k}}\left|E_{N, \varepsilon_{k}, \varepsilon_{k+1}}(\tilde{f})(x)\right|^{2}\right)^{\frac{1}{2}}\right\|_{L^{p}(\mathbb{T})} & \\
& \preceq \frac{1}{N}\left\|\left(\sum_{k=1}^{\infty}\left(t_{k}-t_{k+1}\right)^{2}\right)^{\frac{1}{2}}\right\|_{L^{p}(\mathbb{T})} \preceq \frac{1}{N} .
\end{aligned}
$$

Therefore we obtain that, as $N \rightarrow \infty$,

$$
\left\|\left(\sum_{k=1}^{\infty} \sup _{t_{k+1} \leq \varepsilon_{k+1}<\varepsilon_{k} \leq t_{k}}\left|E_{N, \varepsilon_{k}, \varepsilon_{k+1}}(\tilde{f})(x)\right|^{2}\right)^{\frac{1}{2}}\right\|_{L^{p}(\mathbb{T})}=o(1) .
$$

Similarly, we have for $\varrho>2$,

$$
\begin{aligned}
& \left\|\sup _{\left(\varepsilon_{k}\right) \searrow 0}\left(\sum_{k=1}^{\infty}\left|E_{N, \varepsilon_{k}, \varepsilon_{k+1}}(\tilde{f})(x)\right|^{\varrho}\right)^{\frac{1}{\varrho}}\right\|_{L^{p}(\mathbb{T})} \\
& \preceq \frac{1}{N}\left\|\sup _{\left(\varepsilon_{k}\right) \searrow 0}\left(\sum_{k=1}^{\infty}\left(\varepsilon_{k}-\varepsilon_{k+1}\right)^{\varrho}\right)^{\frac{1}{\varrho}}\right\|_{L^{p}(\mathbb{T})} \preceq \frac{1}{N}\left\|\sup _{\left(\varepsilon_{k}\right) \searrow 0}\left(\sum_{k=1}^{\infty}\left(\varepsilon_{k}-\varepsilon_{k+1}\right)\right)\right\|_{L^{p}(\mathbb{T})} \preceq \frac{1}{N} .
\end{aligned}
$$

Thus we obtain that, as $N \rightarrow \infty$,

$$
\left\|\sup _{\left(\varepsilon_{k}\right) \searrow 0}\left(\sum_{k=1}^{\infty}\left|E_{N, \varepsilon_{k}, \varepsilon_{k+1}}(\tilde{f})(x)\right|^{\varrho}\right)^{\frac{1}{\varrho}}\right\|_{L^{p}(\mathbb{T})}=o(1) .
$$

Proof of Theorem 1.2. With the previous notation, by the definition of $\widetilde{H}_{\varepsilon}(\tilde{f})$ in (2), we rewrite $\widetilde{H}_{\varepsilon_{k}} \tilde{f}-\widetilde{H}_{\varepsilon_{k+1}} \tilde{f}$ as

$$
\begin{aligned}
\widetilde{H}_{\varepsilon_{k}} \tilde{f}(x)-\widetilde{H}_{\varepsilon_{k+1}} \tilde{f}(x)=\frac{1}{\pi} \int_{|t|<\frac{1}{2}} \tilde{f}(x-t)\left(\widetilde{\mathfrak{R}}_{\varepsilon_{k}}(t)-\widetilde{\mathfrak{R}}_{\varepsilon_{k+1}}(t)\right) d t \\
+\frac{1}{\pi} \int_{|t|<\frac{1}{2}} \tilde{f}(x-t)\left(r_{\varepsilon_{k+1}}(t)-r_{\varepsilon_{k}}(t)\right) d t,
\end{aligned}
$$

with the help of Lemma 2.1.

Recall again that $\chi_{A}$ is the characteristic function of the set $|t|>1$. It is easy to see that for $\varepsilon_{k+1}<\varepsilon_{k}<|t| \leq \frac{1}{2}$, we have that for all $j \in \mathbb{Z} \backslash\{0\}$,

$$
\chi_{A}\left(\frac{t+j}{\varepsilon_{k+1}}\right)=\chi_{A}\left(\frac{t+j}{\varepsilon_{k}}\right)=1 .
$$

This leads to

$$
r_{\varepsilon_{k+1}}(t)-r_{\varepsilon_{k}}(t)=0
$$


It now suffices to consider

$$
\mathbb{O}\left(\widetilde{H}_{*} \tilde{f}\right)(x)=\left(\sum_{k=1}^{\infty} \sup _{t_{k+1} \leq \varepsilon_{k+1}<\varepsilon_{k} \leq t_{k}}\left|\int_{|t|<\frac{1}{2}} \tilde{f}(x-t)\left(\widetilde{\mathfrak{R}}_{\varepsilon_{k}}(t)-\widetilde{\mathfrak{R}}_{\varepsilon_{k+1}}(t)\right) d t\right|^{2}\right)^{\frac{1}{2}} .
$$

By (i) of Lemma 2.2, the basic properties of operators on the torus and Theorem C, we conclude that as $N \rightarrow \infty$,

$$
\begin{aligned}
\left\|\mathbb{O}\left(\widetilde{H}_{*} \tilde{f}\right)\right\|_{L^{p}(\mathbb{T})}^{p} & =\frac{1}{N} \int_{-\frac{N}{2}}^{\frac{N}{2}}\left(\sum_{k=1}^{\infty} \sup _{t_{k+1} \leq \varepsilon_{k+1}<\varepsilon_{k} \leq t_{k}}\left|\Psi\left(\frac{x}{N}\right) \tilde{H}_{\varepsilon_{k}, \varepsilon_{k}+1}(\tilde{f})(x)\right|^{2}\right)^{\frac{p}{2}} d x \\
& \preceq o(1)+\frac{1}{N} \int_{-\frac{N}{2}}^{\frac{N}{2}}\left(\sum_{k=1}^{\infty} \sup _{t_{k+1} \leq \varepsilon_{k+1}<\varepsilon_{k} \leq t_{k}}\left|H_{\varepsilon_{i}, \varepsilon_{i}+1}\left(\Psi^{N} \tilde{f}\right)(x)\right|^{2}\right)^{\frac{p}{2}} d x \\
& \preceq o(1)+\frac{1}{N} \int_{\mathbb{R}}\left|\Psi\left(\frac{x}{N}\right) \tilde{f}(x)\right|^{p} d x \preceq\|\tilde{f}\|_{L^{p}(\mathbb{T})}+o(1) .
\end{aligned}
$$

We next show that the oscillation operator $\mathscr{O}\left(\widetilde{H}_{*}\right)$ is of weak type $(1,1)$, that is, for any $\lambda>0$,

$$
\left|\left\{x \in \mathbb{T}:\left|\mathscr{O}\left(\widetilde{H}_{*} \tilde{f}\right)(x)\right|>\lambda\right\}\right| \leq \frac{c}{\lambda}\|\tilde{f}\|_{L^{1}(\mathbb{T})} .
$$

By the basic properties of operators on the torus, we find that for $N \in \mathbb{Z}^{+}$,

$$
\begin{aligned}
\left|\left\{x \in\left[-\frac{1}{2}, \frac{1}{2}\right):\left|\mathscr{O}\left(\widetilde{H}_{*} \tilde{f}\right)(x)\right|>\lambda\right\}\right| & =N^{-1}\left|\left\{x \in\left[-\frac{N}{2}, \frac{N}{2}\right):\left|\mathscr{O}\left(\widetilde{H}_{*} \tilde{f}\right)(x)\right|>\lambda\right\}\right| \\
& =N^{-1}\left|\left\{|x| \leq \frac{N}{2}:\left|\Psi\left(\frac{x}{N}\right) \mathscr{O}\left(\widetilde{H}_{*} \tilde{f}\right)(x)\right|>\lambda\right\}\right| .
\end{aligned}
$$

As in the proofs of (15) and (16), we know that $E_{N, \varepsilon_{k}, \varepsilon_{k+1}}(\tilde{f})(x) \rightarrow 0$ uniformly in $x$ as $N \rightarrow \infty$. For any $\lambda_{1}$ such that $0<\lambda_{1}<\lambda$, choose $N$ large enough that

$$
\left|\left\{x \in\left[-\frac{1}{2}, \frac{1}{2}\right):\left|\mathscr{O}\left(\tilde{H}_{*} \tilde{f}\right)(x)\right|>\lambda\right\}\right| \leq N^{-1}\left|\left\{x \in \mathbb{R}:\left|\mathscr{O}\left(H_{*}\left(\Psi^{N} \tilde{f}\right)\right)(x)\right|>\lambda-\lambda_{1}\right\}\right| .
$$

Theorem $\mathrm{C}$ implies that the last term above can be controlled by

$$
\frac{c N^{-1}}{\lambda-\lambda_{1}}\left\|\Psi^{N} \tilde{f}\right\|_{L^{1}(\mathbb{R})}=\frac{c N^{-1} N}{\lambda-\lambda_{1}}\|\tilde{f}\|_{L^{1}(\mathbb{T})}=\frac{c}{\lambda-\lambda_{1}}\|\tilde{f}\|_{L^{1}(\mathbb{T})} .
$$

Since $\lambda_{1}>0$ is arbitrary, we get the desired result. This completes the proof of Theorem 1.2.

Proof of Theorem 1.3. Using the same argument as in Theorem 1.2, it is enough to study

$$
\mathbb{V}_{\varrho}\left(\widetilde{H}_{*} \tilde{f}\right)(x)=\sup _{\left(\varepsilon_{k}\right) \searrow 0}\left(\sum_{k=1}^{\infty}\left|\int_{|t|<\frac{1}{2}} \tilde{f}(x-t)\left(\widetilde{\mathfrak{R}}_{\varepsilon_{k}}(t)-\widetilde{\mathfrak{R}}_{\varepsilon_{k+1}}(t)\right) d t\right|^{\varrho}\right)^{\frac{1}{\varrho}} .
$$

Now by checking the proof for the oscillation operator $\mathscr{O}\left(\widetilde{H}_{*}\right)$, it suffices to show

$$
\left\|\mathbb{V}_{\varrho}\left(\widetilde{H}_{*} \tilde{f}\right)\right\|_{L^{p}(\mathbb{T})} \preceq\|\tilde{f}\|_{L^{p}(\mathbb{T})} .
$$


Write

$$
\begin{aligned}
& H_{\varepsilon_{k}, \varepsilon_{k}+1}(f)(x)=H_{\varepsilon_{k}} f(x)-H_{\varepsilon_{k+1}} f(x), \\
& \widetilde{H}_{\varepsilon_{k}, \varepsilon_{k}+1}(\tilde{f})(x)=\left(\widetilde{\mathfrak{R}}_{\varepsilon_{k}}-\widetilde{\mathfrak{R}}_{\varepsilon_{k+1}}\right) * \tilde{f}(x) .
\end{aligned}
$$

For any large integer $N$, we define the error term

$$
E_{N, \varepsilon_{k}, \varepsilon_{k+1}}(\tilde{f})(x)=\Psi(x / N) \tilde{H}_{\varepsilon_{k}, \varepsilon_{k}+1}(\tilde{f})-H_{\varepsilon_{k}, \varepsilon_{k}+1}\left(\Psi^{N} \tilde{f}\right)(x) .
$$

Using (ii) of Lemma 2.2, we obtain that as $N \rightarrow \infty$,

$$
\left\|\sup _{\left(\varepsilon_{k}\right) \searrow 0}\left(\sum_{k=1}^{\infty}\left|E_{N, \varepsilon_{k}, \varepsilon_{k+1}}(\tilde{f})(x)\right|^{\varrho}\right)^{\frac{1}{\varrho}}\right\|_{L^{p}(\mathbb{T})}=o(1) .
$$

Finally, applying Theorem D, analogously to the proof of Theorem 1.2 we obtain

$$
\begin{aligned}
\left\|\mathbb{V}_{\varrho}\left(\tilde{H}_{*} \tilde{f}\right)\right\|_{L^{p}(\mathbb{T})}^{p} & =\frac{1}{N} \int_{-\frac{N}{2}}^{\frac{N}{2}} \sup _{\left(\varepsilon_{k}\right) \searrow 0}\left(\sum_{k=1}^{\infty}\left|\Psi\left(\frac{x}{N}\right) \widetilde{H}_{\varepsilon_{k}, \varepsilon_{k}+1}(\tilde{f})(x)\right|^{\varrho}\right)^{\frac{p}{\varrho}} d x \\
& \left.\preceq o(1)+\left.\frac{1}{N} \int_{\mathbb{R}\left(\varepsilon_{k}\right) \searrow 0} \sup _{k=1}\left(\sum_{\varepsilon_{k}, \varepsilon_{k}+1}^{\infty} \mid \Psi^{N} \tilde{f}\right)(x)\right|^{\varrho}\right)^{\frac{p}{\varrho}} d x \\
& \preceq o(1)+\frac{1}{N} \int_{\mathbb{R}}\left|\Psi\left(\frac{x}{N}\right) \tilde{f}(x)\right|^{p} d x \preceq\|\tilde{f}\|_{L^{p}(\mathbb{T})}+o(1) .
\end{aligned}
$$

Letting $N \rightarrow \infty$, we conclude that the variation operator $\mathscr{V}_{\varrho}\left(\widetilde{H}_{*}\right)$ is of strong type $(p, p)$ for $1<p<\infty$.

The same argument as in the proof of Theorem 1.2 works for the weak type $(1,1)$ for the variation operator $\mathscr{V}_{\varrho}\left(\widetilde{H}_{*}\right)$. We omit the details.

Proof of Theorem 1.4. The proof of Theorem 1.4 is similar to that of Theorem 1.3. The only change is to consider two different cases: $p^{\prime}>2$ and $p^{\prime} \leq 2$ in place of the symmetric differentiation operator used above. We leave the details to the interested reader.

\section{Extension to Riesz transforms}

In this section we study the (maximal) bilinear Riesz transforms as $n$-dimensional extensions.

We start with the maximal bilinear singular integral with rough kernel

$$
T_{\Omega}^{*}(f, g)(x)=\sup _{\varepsilon>0}\left|T_{\Omega, \varepsilon}(f, g)(x)\right|,
$$

where $T_{\Omega, \varepsilon}$ is the truncated bilinear operator defined by

$$
T_{\Omega, \varepsilon}(f, g)(x)=\int_{|y|>\varepsilon} \frac{f(x-y) g(x+y)}{|y|^{n}} \Omega\left(y^{\prime}\right) d y \quad \text { for } \varepsilon>0 .
$$


Following the standard rotation method by Calderón and Zygmund (see also [Fan and Zhao $\geq 2016$; Grafakos and Torres 2002]), we have the following result on $\mathbb{R}^{n}$. Theorem 3.1. Let $1<q, r \leq \infty, 1 \leq p<\infty$ and $\frac{1}{p}=\frac{1}{q}+\frac{1}{r}$. If $\Omega \in L^{\infty}\left(S^{n-1}\right)$ is an odd function, then

$$
\left\|T_{\Omega}^{*}(f, g)\right\|_{L^{p}\left(\mathbb{R}^{n}\right)} \preceq\|f\|_{L^{q}\left(\mathbb{R}^{n}\right)}\|g\|_{L^{r}\left(\mathbb{R}^{n}\right)} .
$$

If $\Omega(x)=x_{j} /|x|, j=1,2, \ldots, n$, then (7) and (17) are reduced to the bilinear Riesz transforms and their maximal operators in Euclidean space $\mathbb{R}^{n}$ :

$$
\begin{aligned}
& R_{j}(f, g)(x)=C_{n} \lim _{\varepsilon \rightarrow 0} \int_{|y|>\varepsilon} f(x-y) g(x+y) \frac{y_{j}}{|y|^{n+1}} d y, \\
& R_{j}^{*}(f, g)(x)=C_{n} \sup _{\varepsilon>0}\left|\int_{|y|>\varepsilon} f(x-y) g(x+y) \frac{y_{j}}{|y|^{n+1}} d y\right|, \quad 1 \leq j \leq n,
\end{aligned}
$$

where $y_{j}$ is the $j$-th component of $y$ and $C_{n}=\Gamma((n+1) / 2) \pi^{-(n+1) / 2}$.

Corollary 3.2. Let $1<q, r \leq \infty, 1 \leq p<\infty$ and $\frac{1}{p}=\frac{1}{q}+\frac{1}{r}$. Then

$$
\left\|R_{j}^{*}(f, g)\right\|_{L^{p}\left(\mathbb{R}^{n}\right)} \preceq\|f\|_{L^{q}\left(\mathbb{R}^{n}\right)}\|g\|_{L^{r}\left(\mathbb{R}^{n}\right)} .
$$

As an application, we consider analogous operators on the $n$-dimensional torus $\mathbb{T}^{n}=\left[-\frac{1}{2}, \frac{1}{2}\right)^{n}$.

For $C^{\infty}\left(\mathbb{T}^{n}\right)$ functions $\tilde{f}, \tilde{g}$, write their Fourier series

$$
\tilde{f}(x)=\sum_{k_{1} \in \mathbb{Z}^{n}} a_{k_{1}} e^{2 \pi i\left\langle k_{1}, x\right\rangle}, \quad \tilde{g}(x)=\sum_{k_{2} \in \mathbb{Z}^{n}} b_{k_{2}} e^{2 \pi i\left\langle k_{2}, x\right\rangle},
$$

where $\langle\cdot, \cdot\rangle$ denotes the dot product.

Let

$$
Q=\left\{x=\left(x_{1}, x_{2}, \ldots, x_{n}\right) \in \mathbb{R}^{n}:-\frac{1}{2} \leq x_{j}<\frac{1}{2} \text { for } j=1,2, \ldots, n\right\}
$$

be the fundamental cube on which

$$
\int_{\mathbb{T}^{n}} \tilde{f}(x) d x=\int_{Q} \tilde{f}(x) d x
$$

for all functions $\tilde{f}$ on the torus $\mathbb{T}^{n}$. For $N \in \mathbb{Z}^{+}$, let $N Q$ denote a cube with the same center as $Q$ and side length $N$ times the side length of $Q$. Denote by $Q_{\varepsilon}$ the set given by

$$
Q_{\varepsilon}=\{x \in Q:|x|>\varepsilon\} \text { for } 0<\varepsilon<\frac{1}{2} .
$$

Let

$$
E=\left\{x \in \mathbb{R}^{n}:|x|>1\right\}
$$


and $\chi_{E}(x)$ be the characteristic function of $E$. For $1 \leq i \leq n$, let $x_{i}$ and $m_{i}$ be the $i$-th components of $x=\left(x_{1}, \ldots, x_{n}\right)$ and $m=\left(m_{1}, \ldots, m_{n}\right)$, respectively. For any $x \neq 0$, the kernel of the $j$-th Riesz transform on $\mathbb{R}^{n}$ is

$$
K_{j}(x)=\frac{x_{j}}{|x|^{n+1}} .
$$

Then the kernel of the $j$-th Riesz transform on the torus is defined, in the sense of Cauchy principle value, by

$$
\widetilde{K}_{j}(x)=\sum_{m \in \mathbb{Z}^{n}} \frac{x_{j}+m_{j}}{|x+m|^{n+1}} .
$$

We now define the bilinear Riesz transform $\widetilde{R}_{j}$ and its maximal operator $\widetilde{R}_{j}^{*}$ on the torus $\mathbb{T}^{n}$, for $\tilde{f}, \tilde{g} \in C^{\infty}\left(\mathbb{T}^{n}\right)$, by

$$
\begin{aligned}
& \widetilde{R}_{j}(\tilde{f}, \tilde{g})(x)=\lim _{\varepsilon \rightarrow 0} \widetilde{R}_{j, \varepsilon}(\tilde{f}, \tilde{g})(x), \\
& \widetilde{R}_{j}^{*}(\tilde{f}, \tilde{g})(x)=\sup _{0<\varepsilon<1 / 2}\left|\widetilde{R}_{j, \varepsilon}(\tilde{f}, \tilde{g})(x)\right|,
\end{aligned}
$$

where $\widetilde{R}_{j, \varepsilon}$ is defined by

$$
\begin{aligned}
\widetilde{R}_{j, \varepsilon}(\tilde{f}, \tilde{g})(x) & =\int_{Q_{\varepsilon}} \widetilde{K}_{j}(y) \tilde{f}(x-y) \tilde{g}(x+y) d y \\
& =\int_{Q} \widetilde{K}_{j}(y) \chi_{E}\left(\frac{y}{\varepsilon}\right) \tilde{f}(x-y) \tilde{g}(x+y) d y .
\end{aligned}
$$

Our result can be stated as follows:

Theorem 3.3. Let $1<q, r \leq \infty, 1 \leq p<\infty$ and $\frac{1}{p}=\frac{1}{q}+\frac{1}{r}$. Then

$$
\left\|\widetilde{R}_{j}^{*}(\tilde{f}, \tilde{g})\right\|_{L^{p}\left(\mathbb{T}^{n}\right)} \preceq\|\tilde{f}\|_{L^{q}\left(\mathbb{T}^{n}\right)}\|\tilde{g}\|_{L^{r}\left(\mathbb{T}^{n}\right)} .
$$

By checking the proof of Theorem 1.1, it suffices to show an easy lemma to obtain Theorem 3.3.

Lemma 3.4. For $0<\varepsilon<\frac{1}{2}$ and $y \in Q$, we have the estimate

$$
\widetilde{K}_{j}(y) \chi_{E}\left(\frac{y}{\varepsilon}\right)=\frac{1}{\varepsilon^{n}} \sum_{m \in \mathbb{Z}^{n}} K_{j}\left(\frac{y+m}{\varepsilon}\right) \chi_{E}\left(\frac{y+m}{\varepsilon}\right)-\sum_{m \in \mathbb{Z}^{n} \backslash\{0\}} K_{j}(y+m) \chi_{E^{c}}\left(\frac{y}{\varepsilon}\right)
$$

and

$$
\left|\sum_{m \in \mathbb{Z}^{n} \backslash\{0\}} K_{j}(y+m) \chi_{E^{C}}\left(\frac{y}{\varepsilon}\right)\right| \preceq|y| \chi_{E^{C}}\left(\frac{y}{\varepsilon}\right),
$$

where $E^{C}$ is the complement of the set $E$. 
Proof. The first equality above follows the method of Lemma 2.1. Now we estimate the second inequality. Write

$$
D_{j}^{+}=\left\{m \in \mathbb{Z}^{n} \backslash\{0\}: m_{j}>0\right\}, \quad D_{j}^{0}=\left\{m \in \mathbb{Z}^{n} \backslash\{0\}: m_{j}=0\right\},
$$

and

$$
y^{*}=\left(y_{1}, y_{2}, \ldots, y_{j-1},-y_{j}, y_{j+1}, \ldots, y_{n}\right) \text {. }
$$

Then we have

$$
\begin{aligned}
& \sum_{m \in \mathbb{Z}^{n} \backslash\{0\}} \frac{y_{j}+m_{j}}{|y+m|^{n+1}} \chi_{E^{c}}\left(\frac{y}{\varepsilon}\right) \\
& =\chi_{E^{c}}\left(\frac{y}{\varepsilon}\right) \sum_{m \in D_{j}^{+}}\left(\frac{y_{j}+m_{j}}{|y+m|^{n+1}}+\frac{y_{j}-m_{j}}{\left|y^{*}+m\right|^{n+1}}\right)+\chi_{E^{c}}\left(\frac{y}{\varepsilon}\right) \sum_{m \in D_{j}^{0}} \frac{y_{j}}{|y+m|^{n+1}} \\
& =\chi_{E^{C}}\left(\frac{y}{\varepsilon}\right) y_{j} \sum_{m \in D_{j}^{+}}\left(\frac{1}{|y+m|^{n+1}}+\frac{1}{\left|y^{*}+m\right|^{n+1}}\right) \\
& \quad+\chi_{E^{c}}\left(\frac{y}{\varepsilon}\right) \sum_{m \in D_{j}^{+}} m_{j}\left(\frac{1}{|y+m|^{n+1}}-\frac{1}{\left|y^{*}+m\right|^{n+1}}\right) \\
& \quad+\chi_{E^{c}}\left(\frac{y}{\varepsilon}\right) y_{j} \sum_{m \in D_{j}^{0}}\left(\frac{1}{|y+m|^{n+1}}\right) .
\end{aligned}
$$

It is trivial to get that

$$
\left|\chi_{E^{c}}\left(\frac{y}{\varepsilon}\right) \sum_{m \in D_{j}^{0}} \frac{y_{j}}{|y+m|^{n+1}}\right| \preceq \chi_{E^{c}}\left(\frac{y}{\varepsilon}\right)\left|y_{j}\right|
$$

and

$$
\left|\chi_{E^{C}}\left(\frac{y}{\varepsilon}\right) y_{j} \sum_{m \in D_{j}^{+}}\left(\frac{1}{|y+m|^{n+1}}+\frac{1}{\left|y^{*}+m\right|^{n+1}}\right)\right| \preceq \chi_{E^{C}}\left(\frac{y}{\varepsilon}\right)\left|y_{j}\right| .
$$

Using the mean value theorem,

$$
|f(x)-f(y)| \leq \max _{z \in I}|\nabla f(z)||x-y|,
$$

where $I$ is the line segment between $x$ and $y$. This leads to

$$
\left|\chi_{E^{c}}\left(\frac{y}{\varepsilon}\right) \sum_{m \in D_{j}^{+}} m_{j}\left(\frac{1}{|y+m|^{n+1}}-\frac{1}{\left|y^{*}+m\right|^{n+1}}\right)\right| \preceq \chi_{E^{c}}\left(\frac{y}{\varepsilon}\right)\left|y_{j}\right|,
$$

completing the proof.

From the theorem, we obtain the following corollary. 
Corollary 3.5. Let $1<q, r \leq \infty, 1 \leq p<\infty$ and $\frac{1}{p}=\frac{1}{q}+\frac{1}{r}$. Then

$$
\left\|\widetilde{R}_{j}(\tilde{f}, \tilde{g})\right\|_{L^{p}\left(\mathbb{T}^{n}\right)} \preceq\|\tilde{f}\|_{L^{q}\left(\mathbb{T}^{n}\right)}\|\tilde{g}\|_{L^{r}\left(\mathbb{T}^{n}\right)} .
$$

This corollary corresponds to a result by Blasco and Gillespie [2009, Theorem 1.12] which says that the bilinear Riesz transform $R_{j}$ is bounded from $L^{q}\left(\mathbb{R}^{n}\right) \times L^{r}\left(\mathbb{R}^{n}\right)$ to $L^{p}\left(\mathbb{R}^{n}\right)$, provided $1<q, r \leq \infty, 1 \leq p<\infty$ and $\frac{1}{p}=\frac{1}{q}+\frac{1}{r}$.

\section{Final remarks}

We want to further illustrate that our method works for many operators. In this section, we provide another example. For $\varepsilon>0$, define

$$
R_{j, \varepsilon}(f)(x)=C_{n} \int_{|y|>\varepsilon} f(x-y) \frac{y_{j}}{|y|^{n+1}} d y \quad \text { for } j=1,2, \ldots, n .
$$

Gillespie and Torrea [2004] introduced the oscillation, variation and short variation operators of the Riesz transform $R_{j}$ in $\mathbb{R}^{n}$. The definitions of these three operators can be expressed in forms similar to (4), (5) and (6) with $H_{\varepsilon}$ replaced by $R_{j, \varepsilon}$ in place of the symmetric differentiation operator used above. Gillespie and Torrea also established the $L^{p}\left(\mathbb{R}^{n}\right)$-boundedness of these operators for $1<p<\infty$.

For $C^{\infty}\left(\mathbb{T}^{n}\right)$ functions $\tilde{f}$, write their Fourier series

$$
\tilde{f}(x)=\sum_{k \in \mathbb{Z}^{n}} a_{k} e^{2 \pi i\langle k, x\rangle} .
$$

We define the periodic version of $\widetilde{R}_{j, \varepsilon}$ by

$$
\widetilde{R}_{j, \varepsilon}(\tilde{f})(x)=\int_{Q_{\varepsilon}} \widetilde{K}_{j}(y) \tilde{f}(x-y) d y,
$$

where $\widetilde{K}_{j}$ is defined as in (18).

We now define the oscillation operator $\mathscr{O}\left(\widetilde{R}_{j}\right)$ on the torus by

$$
\mathscr{O}\left(\widetilde{R}_{j} \tilde{f}\right)(x)=\left(\sum_{k=1}^{\infty} \sup _{t_{k+1} \leq \varepsilon_{k+1}<\varepsilon_{k} \leq t_{k}}\left|\widetilde{R}_{j, \varepsilon_{k}} \tilde{f}(x)-\widetilde{R}_{j, \varepsilon_{k+1}} \tilde{f}(x)\right|^{2}\right)^{\frac{1}{2}}
$$

and the variation operator $\mathscr{V}_{\varrho}\left(\widetilde{R}_{j}\right)$ on the torus by

$$
\mathscr{V}_{\varrho}\left(\widetilde{R}_{j} \tilde{f}\right)(x)=\sup _{\left(\varepsilon_{k}\right) \searrow 0}\left(\sum_{k=1}^{\infty}\left|\widetilde{R}_{j, \varepsilon_{k}} \tilde{f}(x)-\widetilde{R}_{j, \varepsilon_{k+1}} \tilde{f}(x)\right|^{\varrho}\right)^{\frac{1}{\varrho}} .
$$

Define the operator $V_{k}\left(\widetilde{R}_{j}\right)$ on the torus by

$$
V_{k}\left(\widetilde{R}_{j} \tilde{f}\right)(x)=\sup _{\left(\varepsilon_{j}\right) \searrow 0}\left(\sum_{\frac{1}{2^{k}}<\varepsilon_{j+1}<\varepsilon_{j} \leq \frac{1}{2^{k-1}}}\left|\widetilde{R}_{j, \varepsilon_{k}} \tilde{f}(x)-\widetilde{R}_{j, \varepsilon_{k+1}} \tilde{f}(x)\right|^{2}\right)^{\frac{1}{2}},
$$


where the supremum is taken over all decreasing sequences $\left(\varepsilon_{j}\right)$. Define the "short variation operator" on the torus by

$$
S_{V}\left(\widetilde{R}_{j} \tilde{f}\right)(x)=\left(\sum_{k=-\infty}^{\infty}\left(V_{k}\left(\widetilde{R}_{j} \tilde{f}(x)\right)^{2}\right)^{\frac{1}{2}} .\right.
$$

Applying the same techniques as in the proof of Theorem 1.2, we can easily transfer those results in [Gillespie and Torrea 2004] from $\mathbb{R}^{n}$ to the torus $\mathbb{T}^{n}$.

\section{Acknowledgements}

The authors are indebted to the anonymous referees for valuable comments that improved the paper significantly.

\section{References}

[Auscher and Carro 1994] P. Auscher and M. J. Carro, "Transference for radial multipliers and dimension free estimates”, Trans. Amer. Math. Soc. 342:2 (1994), 575-593. MR 1152319 Zbl 0812.42009

[Berkson et al. 2006] E. Berkson, O. Blasco, M. J. Carro, and T. A. Gillespie, "Discretization and transference of bisublinear maximal operators", J. Fourier Anal. Appl. 12:4 (2006), 447-481. MR 2256933 Zbl 1130.42016

[Berkson et al. 2007] E. Berkson, O. Blasco, M. J. Carro, and T. A. Gillespie, "Discretization versus transference for bilinear operators", pp. 265-283 in Banach spaces and their applications in analysis (Oxford, OH, 2006), edited by B. Randrianantoanina and N. Randrianantoanina, Walter de Gruyter, Berlin, 2007. MR 2374713 Zbl 1132.42307

[Blasco and Gillespie 2009] O. Blasco and T. A. Gillespie, "Dimension free estimates for the bilinear Riesz transform”, Collect. Math. 60:3 (2009), 249-259. MR 2560969 Zbl 1187.42006

[Blasco et al. 2005] O. Blasco, M. J. Carro, and T. A. Gillespie, "Bilinear Hilbert transform on measure spaces", J. Fourier Anal. Appl. 11:4 (2005), 459-470. MR 2169476 Zbl 1098.42007

[Bourgain 1989] J. Bourgain, "Pointwise ergodic theorems for arithmetic sets", Inst. Hautes Études Sci. Publ. Math. 69 (1989), 5-45. With an appendix by the author, H. Furstenberg, Y. Katznelson and D. S. Ornstein. MR 1019960 Zbl 0705.28008

[Bourgain 1990] J. Bourgain, "Double recurrence and almost sure convergence", J. Reine Angew. Math. 404 (1990), 140-161. MR 1037434 Zbl 0685.28008

[Campbell et al. 2000] J. T. Campbell, R. L. Jones, K. Reinhold, and M. Wierdl, "Oscillation and variation for the Hilbert transform", Duke Math. J. 105:1 (2000), 59-83. MR 1788042 Zbl 1013.42008

[Coifman and Weiss 1977] R. R. Coifman and G. Weiss, Transference methods in analysis (Lincoln, NE, 1976), CBMS Regional Conference Series in Mathematics 31, American Mathematical Society, Providence, RI, 1977. MR 0481928 Zbl 0377.43001

[Edwards and Gaudry 1977] R. E. Edwards and G. I. Gaudry, Littlewood-Paley and multiplier theory, Ergebnisse der Mathematik und ihrer Grenzgebiete 90, Springer, Berlin, 1977. MR 0618663 Zbl 0467.42001

[Fan and Sato 2001] D. Fan and S. Sato, “Transference on certain multilinear multiplier operators", J. Aust. Math. Soc. 70:1 (2001), 37-55. MR 1808390 Zbl 0984.42006

[Fan and Zhao $\geq 2016$ ] D. Fan and F. Zhao, "Certain bilinear operators on Morrey spaces", preprint. To appear in Hokkaido Math. J. 
[Rubio de Francia 1989] J. L. Rubio de Francia, "Transference principles for radial multipliers", Duke Math. J. 58:1 (1989), 1-19. MR 1016410 Zbl 0676.42009

[Gillespie and Torrea 2004] T. A. Gillespie and J. L. Torrea, "Dimension free estimates for the oscillation of Riesz transforms", Israel J. Math. 141 (2004), 125-144. MR 2063029 Zbl 1072.42011

[Grafakos and Honzík 2006] L. Grafakos and P. Honzík, "Maximal transference and summability of multilinear Fourier series", J. Aust. Math. Soc. 80:1 (2006), 65-80. MR 2212316 Zbl 1102.42007

[Grafakos and Torres 2002] L. Grafakos and R. H. Torres, "Multilinear Calderón-Zygmund theory", Adv. Math. 165:1 (2002), 124-164. MR 1880324 Zbl 1032.42020

[Jones 1994] P. W. Jones, "Bilinear singular integrals and maximal functions", Chapter 10.2, p. 414, in Linear and complex analysis: problem book 3, part I, edited by V. P. Havin and N. K. Nikolski, Lecture Notes in Mathematics 1573, Springer, Berlin, 1994.

[Jones 1997] R. L. Jones, "Ergodic theory and connections with analysis and probability", New York J. Math. 3A (1997), 31-67. MR 1604581 Zbl 0898.28005

[Jones et al. 1998] R. L. Jones, R. Kaufman, J. M. Rosenblatt, and M. Wierdl, "Oscillation in ergodic theory”, Ergodic Theory Dynam. Systems 18:4 (1998), 889-935. MR 1645330 Zbl 0924.28009

[Lacey 2000] M. T. Lacey, "The bilinear maximal functions map into $L^{p}$ for $2 / 3<p \leq 1$ ", Ann. of Math. (2) 151:1 (2000), 35-57. MR 1745019 Zbl 0967.47031

[Lacey and Thiele 1997] M. T. Lacey and C. Thiele, " $L^{p}$ estimates on the bilinear Hilbert transform for $2<p<\infty$ ”, Ann. of Math. (2) 146:3 (1997), 693-724. MR 1491450 Zbl 0914.46034

[de Leeuw 1965] K. de Leeuw, “On $L_{p}$ multipliers", Ann. of Math. (2) 81 (1965), 364-379. MR 0174937 Zbl 0171.11803

[Stein and Weiss 1971] E. M. Stein and G. Weiss, Introduction to Fourier analysis on Euclidean spaces, Princeton Mathematical Series 32, Princeton University Press, 1971. MR 0304972 Zbl 0232. 42007

[Varadarajan 2007] V. S. Varadarajan, "Euler and his work on infinite series", Bull. Amer. Math. Soc. (N.S.) 44:4 (2007), 515-539. MR 2338363 Zbl 1135.01010

Received January 25, 2015.

DASHAN FAN

DEPARTMENT OF MATHEMATICAL SCIENCES

UNIVERSITY OF WISCONSIN-MILWAUKEE

MiLWAUKEE, WI 53201

UNited STATES

fan@uwm.edu

HUOXIONG WU

SCHOOL OF Mathematical SCIENCES

XIAMEN UNIVERSITY

361005 FUJIAN

CHINA

huoxwu@xmu.edu.cn

FAYOU ZHAO

DEPARTMENT OF MATHEMATICS

SHANGHAI UNIVERSITY

200444 SHANGHAI

CHINA

fyzhao@shu.edu.cn 


\title{
PACIFIC JOURNAL OF MATHEMATICS
}

Founded in 1951 by E. F. Beckenbach (1906-1982) and F. Wolf (1904-1989)

$$
\text { msp.org/pjm }
$$

\section{EDITORS}

\author{
Don Blasius (Managing Editor) \\ Department of Mathematics \\ University of California \\ Los Angeles, CA 90095-1555 \\ blasius@math.ucla.edu
}

\author{
Paul Balmer \\ Department of Mathematics \\ University of California \\ Los Angeles, CA 90095-1555 \\ balmer@math.ucla.edu \\ Robert Finn \\ Department of Mathematics \\ Stanford University \\ Stanford, CA 94305-2125 \\ finn@math.stanford.edu \\ Sorin Popa \\ Department of Mathematics \\ University of California \\ Los Angeles, CA 90095-1555 \\ popa@math.ucla.edu
}

\author{
Vyjayanthi Chari \\ Department of Mathematics \\ University of California \\ Riverside, CA 92521-0135 \\ chari@math.ucr.edu \\ Kefeng Liu \\ Department of Mathematics \\ University of California \\ Los Angeles, CA 90095-1555 \\ liu@math.ucla.edu \\ Igor Pak \\ Department of Mathematics \\ University of California \\ Los Angeles, CA 90095-1555 \\ pak.pjm@gmail.com \\ Paul Yang \\ Department of Mathematics \\ Princeton University \\ Princeton NJ 08544-1000 \\ yang@math.princeton.edu
}

\section{PRODUCTION}

Silvio Levy, Scientific Editor, production@msp.org

\section{SUPPORTING INSTITUTIONS}

ACADEMIA SINICA, TAIPEI

CALIFORNIA INST. OF TECHNOLOGY

STANFORD UNIVERSITY

UNIV. OF BRITISH COLUMBIA

UNIV. OF CALIFORNIA, BERKELEY

UNIV. OF CALIFORNIA, DAVIS

UNIV. OF CALIFORNIA, LOS ANGELES

UNIV. OF CALIFORNIA, RIVERSIDE

UNIV. OF CALIFORNIA, SAN DIEGO

UNIV. OF CALIF., SANTA BARBARA
KEIO UNIVERSITY

MATH. SCIENCES RESEARCH INSTITUTE

NEW MEXICO STATE UNIV.

OREGON STATE UNIV.
Daryl Cooper

Department of Mathematics

University of California

Santa Barbara, CA 93106-3080 cooper@math.ucsb.edu

Jiang-Hua Lu

Department of Mathematics

The University of Hong Kong

Pokfulam Rd., Hong Kong

jhlu@maths.hku.hk

$$
\text { Jie Qing }
$$

Department of Mathematics

University of California

Santa Cruz, CA 95064

qing@ cats.ucsc.edu

\author{
UNIV. OF CALIF., SANTA CRUZ \\ UNIV. OF MONTANA \\ UNIV. OF OREGON \\ UNIV. OF SOUTHERN CALIFORNIA \\ UNIV. OF UTAH \\ UNIV. OF WASHINGTON \\ WASHINGTON STATE UNIVERSITY
}

These supporting institutions contribute to the cost of publication of this Journal, but they are not owners or publishers and have no responsibility for its contents or policies.

See inside back cover or msp.org/pjm for submission instructions.

The subscription price for 2016 is US $\$ 440 /$ year for the electronic version, and \$600/year for print and electronic.

Subscriptions, requests for back issues and changes of subscriber address should be sent to Pacific Journal of Mathematics, P.O. Box 4163, Berkeley, CA 94704-0163, U.S.A. The Pacific Journal of Mathematics is indexed by Mathematical Reviews, Zentralblatt MATH, PASCAL CNRS Index, Referativnyi Zhurnal, Current Mathematical Publications and Web of Knowledge (Science Citation Index).

The Pacific Journal of Mathematics (ISSN 0030-8730) at the University of California, c/o Department of Mathematics, 798 Evans Hall \#3840, Berkeley, CA 94720-3840, is published twelve times a year. Periodical rate postage paid at Berkeley, CA 94704, and additional mailing offices. POSTMASTER: send address changes to Pacific Journal of Mathematics, P.O. Box 4163, Berkeley, CA 94704-0163.

PJM peer review and production are managed by EditFLOW ${ }^{\circledR}$ from Mathematical Sciences Publishers.

PUBLISHED BY

\section{I. mathematical sciences publishers}

nonprofit scientific publishing

http://msp.org/

(C) 2016 Mathematical Sciences Publishers 


\section{PACIFIC JOURNAL OF MATHEMATICS}

Volume $284 \quad$ No. $2 \quad$ October 2016

Spherical CR Dehn surgeries

Miguel ACOsta

Degenerate flag varieties and Schubert varieties: a characteristic free approach

283

Giovanni Cerulli Irelli, Martina LANini and Peter

LITTELMANN

Solitons for the inverse mean curvature flow

Gregory Drugan, HoJoo LEE and GLEN WHEELER

Bergman theory of certain generalized Hartogs triangles

\section{LUKE D. EDHOLM}

Transference of certain maximal Hilbert transforms on the torus

DASHAN FAN, HUOXIONG WU and FAYOU ZHAO

The Turaev and Thurston norms

STEFAN FriedL, DANIEL S. Silver and Susan G. WiLLiams

A note on nonunital absorbing extensions

JAMES GABE

On nonradial singular solutions of supercritical biharmonic equations

Zongming GuO, JUNCHENG WeI and Wen YANG

Natural commuting of vanishing cycles and the Verdier dual

\section{DAVID B. MASSEY}

The nef cones of and minimal-degree curves in the Hilbert schemes of points on certain surfaces

ZHENBo QIN and YUPING TU

Smooth approximation of conic Kähler metric with lower Ricci curvature bound

\section{LIANGMING SHEN}

Maps from the enveloping algebra of the positive Witt algebra to regular algebras

Susan J. SierRa and Chelsea Walton 